\title{
Technical note: Evaluation of fine needle aspiration cytology for the diagnosis of fatty liver in dairy cattle
}

\author{
P. Melendez, ${ }^{* 1}$ M. Whitney, ${ }^{*}$ F. Williams, ${ }^{*}$ P. Pinedo, $†$ D. Manriquez,† S. G. Moore $\ddagger$ M. C. Lucy,, P. Pithua, ${ }^{*}$ \\ and S. E. Poock* \\ ${ }^{*}$ College of Veterinary Medicine, University of Missouri, Columbia 65211 \\ †Department of Animal Sciences, Colorado State University, Fort Collins 80521 \\ ‡Division of Animal Sciences, University of Missouri, Columbia 65211
}

\section{ABSTRACT}

Fatty liver is a common condition affecting dairy cattle during the periparturient period, characterized by a pathological accumulation of triglycerides (TG) in the hepatocytes. The objective of this study was to evaluate the diagnostic potential of fine needle aspiration cytology in fresh liver specimens using liver TG concentrations as a gold standard. Fifty-seven liver samples from Holstein cows were collected during processing at a slaughterhouse. Tissue and fine needle aspirate samples were obtained from the parietal upper portion of the caudate lobe. Two samples of liver tissue were collected with a 16 gauge $\times 15 \mathrm{~cm}$ biopsy needle for histological and TG concentration assessment. A third sample was collected for cytology using an 18 gauge $\times 5.08 \mathrm{~cm}$ needle. The contents of the needle were transferred to a glass slide, spread, and air-dried. Liver samples were assayed by colorimetry/fluorimetry to determine TG concentrations. Concentrations of TG $<2 \%$ were considered normal. Histological and cytological evaluations were conducted by 2 different pathologists blind to the visual classification. Sensitivity (Se) and specificity (Sp) were calculated. Cytology had a Se and Sp of 73 and $85 \%$, respectively. Histopathology had a Se and Sp of 45.9 and $100 \%$, respectively. The likelihood of having higher scores for histopathology and cytology increased as a function of liver TG content $(\mathrm{mg} / \mathrm{g})$.

Key words: fatty liver, cytology, histology, triglycerides

\section{Technical Note}

Hepatic lipidosis, also known as fatty liver, is a common condition affecting dairy cattle during the periparturient period, characterized by a pathological accumulation of triglycerides (TG) in the hepatocytes

Received June 17, 2017.

Accepted January 14, 2018.

${ }^{1}$ Corresponding author: melendezp@missouri.edu
(Herdt, 1988). This condition develops secondary to the typical negative energy balance experienced by the dairy cow during the early postpartum period, especially in those individuals with a high body condition at parturition. This hepatic infiltration is a consequence of fat mobilization from adipose tissue, which leads to an increase in fatty acids (FA) that are transported to the liver (Herdt, 2000; Bobe et al., 2004; Gerspach et al., 2017). When FA release is severe due to adverse conditions, such as obesity, disease, and poor transition feed management, excess FA in the liver are re-esterified to TG. The rate of liver export of TG as very low density lipoprotein is a slow mechanism in cattle; therefore, the net outcome is a pathological TG accumulation leading to fatty liver syndrome (Herdt, 1988; Bobe et al., 2004).

At present, there is no biochemical diagnostic test that is accurate, fast, and reliable for the diagnosis of this condition (Herdt, 1988; Kalaitzakis et al., 2010). Hepatic TG content can be determined by fat extraction, histopathology, or flotation of liver biopsies obtained in live animals (Herdt, 1988; Bobe et al., 2004). However, liver biopsies are invasive and associated with risks of hemorrhage, infection, and adhesions (Bobe et al., 2004; Swecker, 2014). Another technique for the diagnosis of hepatic lipidosis in cattle is transcutaneous ultrasound; nevertheless, this methodology has low sensitivity (Se) when TG contents are $<10 \%$. This method is therefore more accurate for the diagnosis of severe fatty liver (TG >10\%; Haudum et al., 2011; Rafia et al., 2011; Starke et al., 2011; Weijers et al., 2012).

The use of fine needle aspiration cytology (FNAC) for the diagnosis of fatty liver in cattle and other species has been described (Hoff and Cote, 1996; Weiss and Moritz, 2002); however, it has not been extensively evaluated in dairy cows. Fine needle aspiration cytology is technically feasible in clinical practice, being minimally invasive and inexpensive, and having a rapid turnaround time compared with biopsy (Komemushi et al., 2015). Consequently, the aim of this study was to evaluate the diagnostic potential of FNAC for hepatic lipidosis using liver TG extraction as a gold standard. 
Sampling was conducted from a slaughterhouse located in Green Bay, Wisconsin. Livers were selected according to the following criteria: (1) originated from adult mature cows, and (2) no observable evident lesions, such as abscesses, tumors, cysts, and so on. To obtain variability regarding the TG content of the livers, a visual scoring based on surface color was performed to classify the organs. Considering a 2-sample proportions test, with a difference in probabilities of $50 \%$ and $95 \%$ of confidence and $80 \%$ of power, a total sample size of 38 livers (19 visually abnormal and 19 visually normal) was determined. To obtain enough variability, 3 classes were considered and 19 livers per classes were sampled. Class 1 was defined as a normal liver with a homogeneous maroon color. Class 2 was defined as a liver with moderate yellow-pale color, and class 3 was defined as a liver with an extreme yellow color.

Eligible livers $(\mathrm{n}=57)$ were removed from the processing line and placed on a metal table to proceed with the sampling procedure. Both tissue and needle aspirate samples were obtained from the parietal upper portion of the caudate lobe. This area was sampled because it is the normal section for conducting liver biopsies in live adult cattle (superior part of the 12th right side intercostal space). Two pieces of liver were collected with a biopsy tool (16 gauge $\times 15 \mathrm{~cm}$; Tru-Cut biopsy needle, Jorgensen Laboratories, Loveland, CO). One piece was stored in a plastic tube with $10 \%$ formalin for histologic evaluation. The second piece was stored in a tube without preservation medium and placed immediately in a plastic cooler with dry ice for TG extraction. A third sample for cytology was obtained using an 18 gauge $\times$ $5.03 \mathrm{~cm}$ needle. Half of the needle was introduced immediately next to the area where the liver biopsies were obtained followed by aspiration with a $20-\mathrm{mL}$ syringe. The content collected inside of the needle was spread on a glass slide and air-dried.

Biopsy samples stored in formalin and liver smears were submitted for histologic and cytologic evaluation, respectively, at the College of Veterinary Medicine, University of Missouri. Liver samples for TG extraction were frozen at $-80^{\circ} \mathrm{C}$ until the analyses were performed.

Triglyceride extraction was used as the gold standard for comparison with both microscopic techniques. Approximately $100 \mathrm{mg}$ of liver tissue was obtained from biopsies. The amount of TG per gram of wet tissue was measured using a TG quantification kit (ab65336; Abcam, Cambridge, MA) that uses a colorimetric methodology. Tissue sample processing was according to the manufacturer's instructions (http://www.abcam .com/triglyceride-assay-kit-quantification-ab65336 .html). The liver samples were weighed and placed in $1 \mathrm{~mL}$ of in 5\% NP40 in water and homogenized by using a Tissue Tearor homogenizer (Biospec Products
Inc., Bartlesville, OK). The samples were then heated to 80 to $100^{\circ} \mathrm{C}$ in a water bath for approximately $5 \mathrm{~min}$, cooled to room temperature, and then heated again. The homogenates were then centrifuged at 20,000 $\times g$ for $2 \mathrm{~min}$ at $23^{\circ} \mathrm{C}$ and the supernatant was diluted 10 fold in water. Ten microliters of the diluted supernatant was assayed. In this assay, TG are converted to free FA and glycerol; the glycerol is then oxidized to generate a product which reacts with the probe to generate color $(\lambda=570 \mathrm{~nm})$ and fluorescence (excitation/emission $=$ $535 / 587 \mathrm{~nm}$ ). The assay can detect $2 \mathrm{pmol}$ to $10 \mathrm{nmol}$ (2 $\mu M$ to $10 \mathrm{~m} M$ range) of TG. Sample and background assays were performed and background was subtracted from the sample values. The assay gave results in nanomoles per well, which was corrected for sample dilution. The nanomoles of TG were converted to mass of TG by using the equation $1 \mathrm{nmol}=850 \mathrm{ng}$ of TG. The weight of TG was then expressed as a percentage of the wet tissue weight of the starting sample.

Normal livers were defined as having a TG content of $<2 \%$. Fatty liver was defined as TG content of $\geq 2 \%$ (Petit et al., 2007; Gross et al., 2013; Crookenden et al., 2016). This assumption was considered because subclinical molecular and pathological changes of the liver start as early as with a content of TG $\geq 2 \%$ (Gross et al., 2013; Crookenden et al., 2016). Biopsy samples for histopathology were fixed in paraffin wax and stained with hematoxylin and eosin. The evaluator was a trained anatomic pathologist from the College of Veterinary Medicine, University of Missouri, board certified by the American College of Veterinary Pathologists. The evaluator was blind to the visual classification score of samples. Microscopic assessment was based on a score of 0 to 4 related to cytoplasm fat infiltration (Figure 1). Histologically, hepatocellular lipid appears as a clear round vacuole. The vacuoles are clear because lipid is removed in routine processing of tissue for histopathology, such as the stain with hematoxylin and eosin. In addition, glycogen accumulation in the liver can lead to variable degrees of pallor and swelling. Microscopically, hepatocytes are swollen with lacy vacuolated cytoplasm. Unlike lipid vacuoles, glycogen vacuoles are poorly defined and hepatocyte nuclei are not displaced to the periphery of the cell (Brown et al., 2017).

Slides with needle aspirate smears were processed in the clinical pathology laboratory of the Veterinary Medical Diagnostic Laboratory. Slides were stained with a modified Wright-Giemsa stain using an automated slide stainer (Hema-tek model 4488CE, Siemens Healthcare Diagnostics Inc., Tarrytown, NY) and examined and classified by a clinical pathologist board certified by the American College of Veterinary Pathologists. The clinical pathologist was blind to the visual classification score of the samples and histopathologic 
evaluation. Microscopic examination was used to assign each sample a score of 0 to 4 based on the degree of intracellular lipid accumulation in several representative clusters of hepatocytes. Scores are described in Figure 2 and are based on a scoring system proposed by Hoff and Cote (1996). In cytology specimens, lipid vacuolation consists of discrete round vacuoles. When numerous, they displace the nucleus. Nonlipid vacuolar change appears as wispy cytoplasmic clearing and the nucleus is unaffected (Arndt and Shelly, 2014).

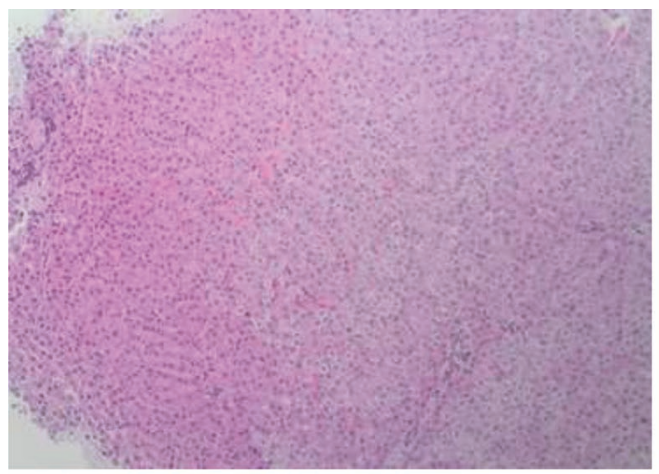

\section{Score 0}

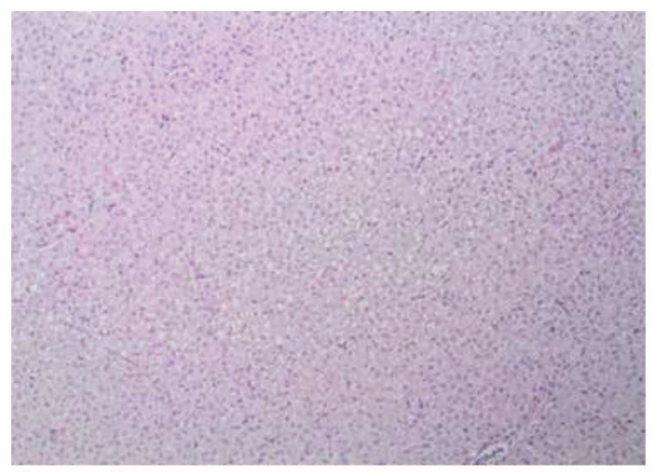

Score 2

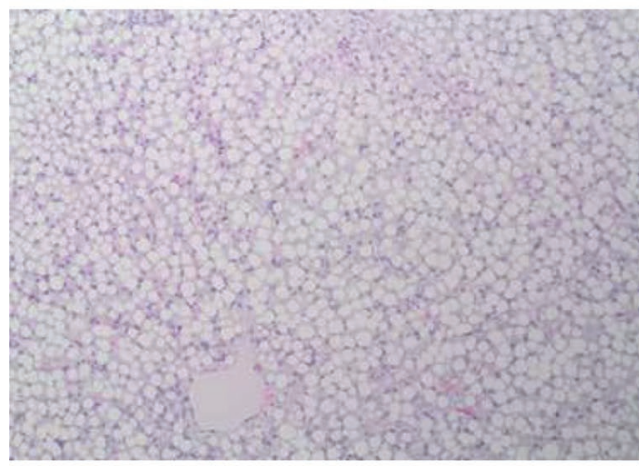

Score 4
Sensitivity, specificity (Sp), positive predictive value, and negative predictive value were calculated. To accomplish this calculation, a dichotomization of the variables was conducted. Triglyceride content was classified as $<2 \%$ (normal) or $\geq 2 \%$ (abnormal). For histopathology, and based on the evaluator expertise, the scores 0 and 1 were classified as normal and scores 2,3 , and 4 as abnormal. For cytology, and based on the clinical pathologist expertise, scores 0 and 1 were classified as normal, and scores 2,3 , and 4 as abnormal.

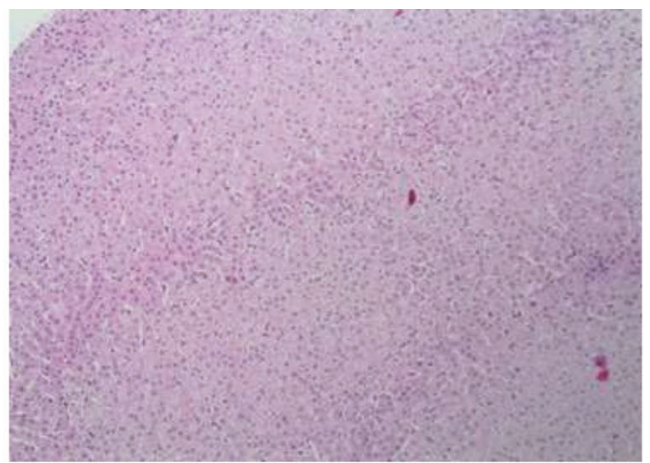

\section{Score 1}

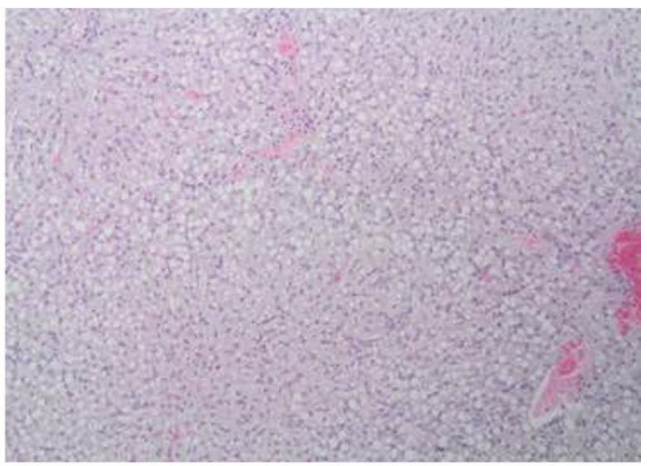

Score 3

Figure 1. Illustration of the scores considered for histopathology. For the analyses, scores 0 and 1 were considered normal and scores 2,3 , and 4 were considered abnormal. Stained with hematoxylin and eosin, original magnification $\times 100$. 
The analysis was conducted using the PROC FREQ in SAS 9.4 (SAS Institute Inc., Cary, NC). In addition, the agreement between tests (cytology vs. TG content, histopathology vs. TG content, and cytology vs. histopathology) was determined by calculation of the kappa statistics using the PROC SURVEYFREQ of SAS 9.4. A cumulative logit regression analysis for ordinal data (cytology and histopathology scores) and liver TG content treated as continuous variable was conducted. Predicted probabilities for scores of both techniques were plotted. Statistical analysis was conducted using the PROC LOGISTIC of SAS 9.4 (SAS Institute Inc.).

Results for Se, Sp, positive predictive value, and negative predictive value are shown in Table 1. Cytol- ogy had a Se and Sp of 73 and $85 \%$, respectively. Histopathology had a Se and Sp of 45.9 and 100\%, respectively. Kappa statistics are shown in Table 2. Kappa statistics, measuring the agreement between cytology and TG content, histopathology and TG content, and cytology and histopathology were $0.53,0.37$, and 0.35 , respectively.

For the cumulative logit analysis (Table 3; Figures 3 and 4 ), the interpretation was that for each $1 \%$ of increment in TG liver content the odds of having a higher cytological score was 1.38 times more likely than having a lower score. For histopathology, the odds of having a higher score was 1.45 times more likely than having a lower score. The same results are shown in the graphs.

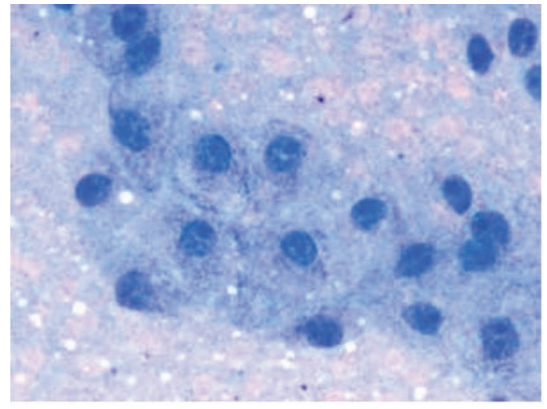

Score 0

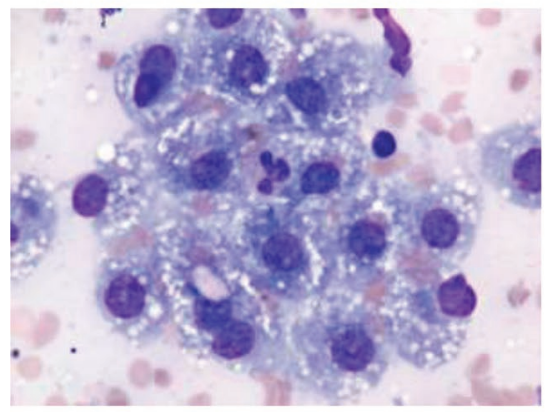

Score 2

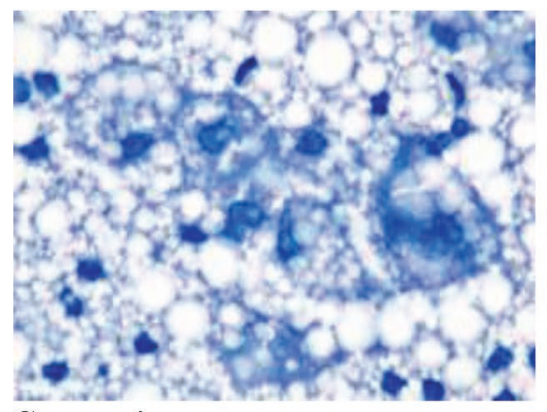

Score 4

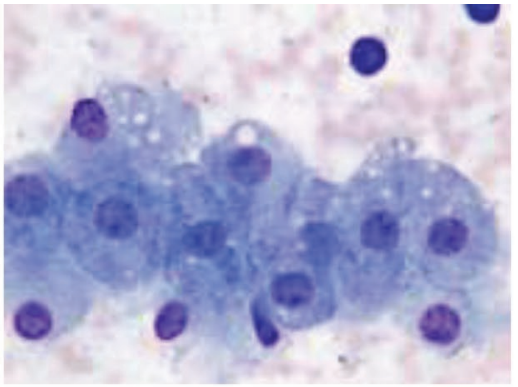

Score 1

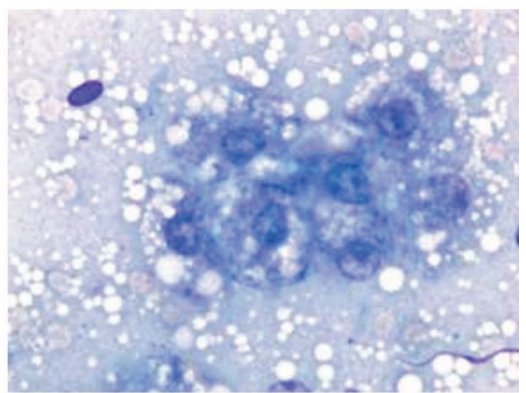

Score 3

Figure 2. Scores for fine needle aspiration cytology. For the analyses, scores 0 and 1 were considered normal and scores 2 , 3 , and 4 were considered abnormal. Modified Wright-Giemsa stain, original magnification $\times 1,000$. 
Table 1. Sensitivity, specificity, and positive and negative predictive values for cytology and histopathology using triglyceride concentration in the liver $(\mathrm{n}=57)$ as a gold standard (cutoff of $2 \%$ )

\begin{tabular}{lccccccc}
\hline & \multicolumn{2}{c}{ Cytology } & & \multicolumn{2}{c}{ Histopathology } \\
\cline { 2 - 4 } \cline { 6 - 8 } Item & Estimate & \multicolumn{2}{c}{$95 \%$ CI } & & Estimate & $95 \%$ CI \\
\hline Prevalence (\%) & 65.0 & 51.0 & 77.1 & & 65.0 & 51.0 & 77.1 \\
Sensitivity (\%) & 73.0 & 55.9 & 86.2 & & 45.9 & 29.5 & 63.1 \\
Specificity (\%) & 85.0 & 62.1 & 96.8 & & 100 & 83.2 & 100 \\
Positive predictive value (\%) & 90.0 & 75.7 & 96.3 & & 100 & 80.5 & 100 \\
Negative predictive value (\%) & 63.0 & 49.3 & 74.9 & & 50 & 42.6 & 57.4 \\
\hline
\end{tabular}

Probabilities of lower scores decrease and probabilities of higher scores increase as a function of increment of liver TG content.

Our study aimed to provide more information related to the diagnostic ability of FNAC for hepatic lipidosis in dairy cattle. Fatty liver is a pathological condition that varies in magnitude ranging from a moderate state of fat infiltration (2 to 5\%), to more than $10 \%$ of TG accumulation (Bobe et al., 2004). The use of the cutoff value of $2 \%$ was considered based on the studies published by Gross et al. (2013) and Crookenden et al. (2016), where a liver TG content $>2 \%$ would already show subclinical molecular and pathological changes. Indeed, content of hepatic TG in prepartum cows and well-fed cows around 100 DIM was between 1.8 and $2.5 \%$. After 1 wk postpartum, liver TG content increased over $5 \%$. In addition, the solute carrier family 27, member 1, also known as SLC27A1, which mediates the translocation of long-chain FA across plasma membranes, was low with a liver TG content of $2 \%$, and the expression of SLC27A1 was highest at $1 \mathrm{wk}$ postpartum along with the highest plasma nonesterified fatty acids (NEFA) and liver TG concentration. These findings would support the mRNA expression pattern of SLC27A1 during the negative energy balance in both early lactation and in feed restricted animals at 100 DIM, as an indicator of enhanced uptake of plasma NEFA and TG accumulation in the liver (Gross et al., 2013). In a more recent study (Crookenden et al., 2016), proteins from circulating exosomes were compared between cows, at $1 \mathrm{wk}$ postpartum, with higher risk of metabolic dysfunction $(1.8 \mathrm{mEq} / \mathrm{L}$ of NEFA; 1.0 $\mathrm{mmol} / \mathrm{L}$ of BHB; and $9.0 \%$ hepatic TG) and lower risk of metabolic dysfunction $(0.66 \mathrm{mEq} / \mathrm{L}$ of NEFA; 0.45
mmol/L of BHB; and $1.8 \%$ hepatic TG). The authors described that exosomes are small, membrane-bound extracellular vesicles (ECV; 40-100 $\mathrm{nm}$ in diameter) exocytosed by cells, containing components from the propagating cell such as proteins, lipids, and nucleic acids. Aberrant cellular function (particularly pathologic conditions) can alter the rate of release of ECV as well as their protein composition, such that the contents reflect the physiological state of the originating cell. In addition, as ECV are transported in blood, they may function as potential biomarkers of disease state. Due to this relationship, exosomes isolated from cows at risk of metabolic dysfunction during the transition period may exhibit an altered protein composition and could be a useful biomarker of health. Cows in the high risk category were chosen because their metabolic profile reflected an extreme tendency to mobilize adipose reserves (high NEFA), an inability of the liver to oxidize mobilized NEFA completely (high BHB), and a failure of the liver to export the resultant very low density lipoprotein to peripheral tissues (high liver TG). A higher concentration of both envoplakin and oncoprotein-induced transcript-3 were elevated in the cows at increased risk of disease compared with cows with low hepatic TG content (1.8\%). These proteins have been identified as important for the liver function. Consequently, the authors of this study proposed that these proteins reflect hepatic steatosis and may be useful biomarkers of a more severe pathological condition before clinical signs are evident, starting to increase as early as with $2 \%$ of hepatic TG content.

However, other studies have shown that pathological changes in the liver, expressed as increased plasma concentration of haptoglobin, may be evident with a

Table 2. Kappa statistics between cytology versus histopathology, histopathology versus liver triglyceride (TG) content, and cytology versus liver TG content

\begin{tabular}{lccc}
\hline Test & $\begin{array}{c}\text { Simple kappa } \\
\text { coefficient }\end{array}$ & $\begin{array}{c}\text { McNemar's test } \\
P \text {-value }\end{array}$ & $95 \%$ CI \\
\hline Cytology vs. histopathology & 0.35 & 0.003 & $0.13-0.56$ \\
Histopathology vs. TG content & 0.37 & 0.0001 & $0.20-0.54$ \\
Cytology vs. TG content & 0.53 & 0.05 & $0.32-0.75$ \\
\hline
\end{tabular}


Table 3. Analysis for the cumulative logit and odd ratio estimates for the prediction scores of cytology and histopathology for each $1 \%$ increment in the content of liver triglycerides (TG)

\begin{tabular}{lcccc}
\hline Effect & $\begin{array}{c}\text { Point estimate } \\
\text { odds ratio }\end{array}$ & $\begin{array}{c}\text { 95\% Wald } \\
\text { confidence limits }\end{array}$ & $\begin{array}{c}\text { Test for null hypothesis } \\
\text { LR }^{1}(P \text {-value })\end{array}$ & $\begin{array}{c}\text { Model fit } \\
\text { AIC }^{2}\end{array}$ \\
\hline TG content for cytology (for each 1\% increment) & 1.38 & 1.18 & 1.62 & $19.7(<0.0001)$ \\
TG content for histopathology (for each 1\% increment) & 1.45 & 1.23 & 1.72 & $24.8(<0.0001)$ \\
\hline
\end{tabular}

${ }^{1} \mathrm{LR}=$ likelihood ratio chi-squared.

${ }^{2} \mathrm{AIC}=$ Akaike's information criterion.

TG content $>2.5 \%$ in primiparous cows and $>4 \%$ in multiparous cows (McCarthy et al., 2016). In addition, in Holstein cattle in Japan, no evidence of apoptosis (expressed as DNA damage and increase of caspase-3 expression) was shown in cows with TG content of $5 \%$, and these changes were evident when liver TG content was $\sim 12 \%$ (Tharwat et al., 2012). Undoubtedly, the hepatic content of TG to which an animal can already show signs of liver damage is relative to several factors, such as the number of lactation, the state of lactation (early or mid-lactation), the physiological state (prepartum, postpartum), genetics or breed, and so on. This is important because by increasing the cutoff value of TG content for hepatic lipidosis diagnosis, the Se of a test is decreased and the Sp is increased. Consequently, the value of $2 \%$ for liver TG content was arbitrarily selected as a cut-off for liver disease based on the early molecular changes reported in the above mentioned studies. Nevertheless, we acknowledge that a $2 \%$ liver content of TG as a cut-off value for hepatic lipidosis is a conservative number, and perhaps a concentration between 2 and $5 \%$ of liver TG, based on previous studies (Jorritsma et al., 2001; Geelen and Wensing, 2006; Gross et al., 2013), is a more represen-

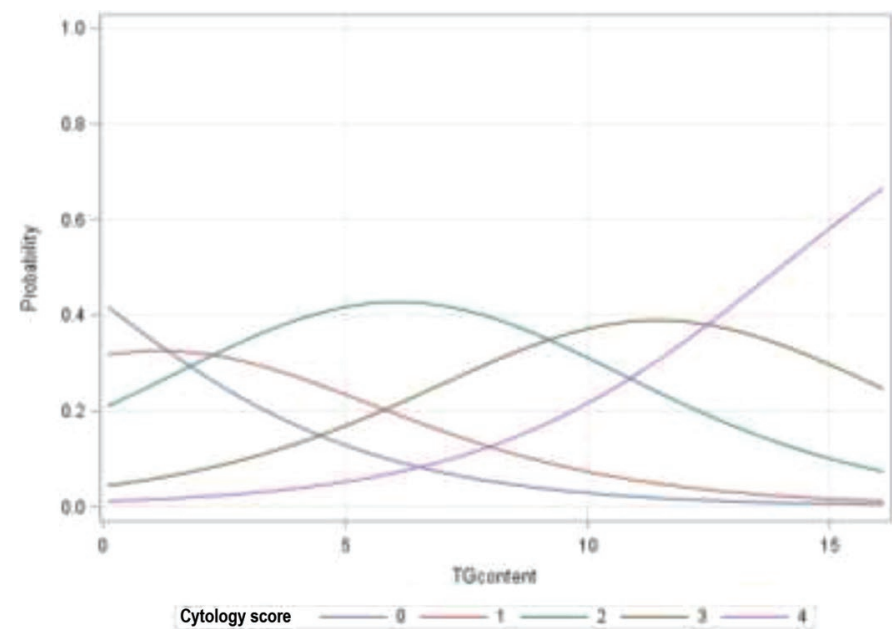

Figure 3. Predicted probabilities for cytology scores as a function of liver triglyceride (TG) content (\%). Color version available online. tative range to establish a cut-off value for fatty liver. These arguments offer an opportunity to investigate a more consistent cut-off value for fatty liver definition in future studies. Perhaps under certain physiological and environmental circumstances $2 \%$ is the value and under other circumstances the concentration might be between a larger range $(2-7 \%)$.

Noninvasive techniques that aid in the diagnosis of fatty liver in dairy cattle include the determination of circulating levels of orinithine carbamoyl transferase $(>25 \mathrm{U} / \mathrm{L})$, glutamate dehydrogenase $(>8.9 \mathrm{U} / \mathrm{L})$; NEFA ( $>1 \mathrm{mmol} / \mathrm{L}$; Kalaitzakis et al., 2010), decreased liver propionate to glucose conversion (McCarthy et al., 2015), and altered transcutaneous ultrasound imaging (TG liver content >10\%; Haudum et al., 2011; Rafia et al., 2011; Starke et al., 2011; Weijers et al., 2012). Unfortunately, these methods are only reliable when the hepatic TG content is $>5 \%$.

One of the findings of this study is that cytology offers an acceptable Se (73\%) and Sp (85\%) using a cut-off value of $2 \%$ for liver TG content. An early diagnosis of mild fatty liver (TG infiltration $2-5 \%$ ) can be helpful to establish the occurrence of this disease and the therapeutic and preventative methods to be used.

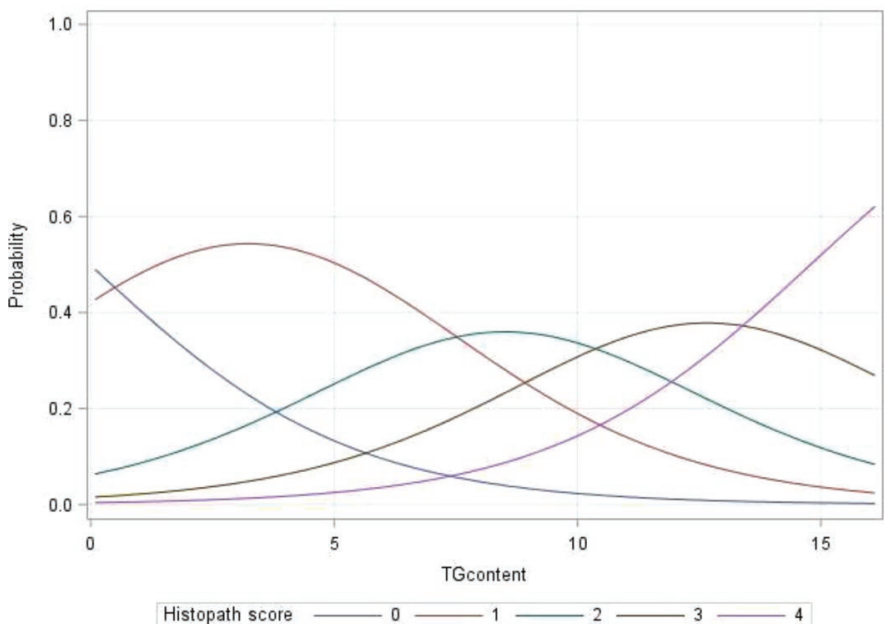

Figure 4. Predicted probabilities for histopathology (histopath) scores as a function of liver triglyceride (TG) content (\%). Color version available online. 
Despite the fact that histopathology has also been considered an effective method in the diagnosis of fatty liver in dairy cattle (Bobe et al., 2004), humans, and other species (Rothuizen and Twedt, 2009; Nalbantoglu and Brunt, 2014), the present study had a higher proportion of false negatives using histopathology (54.1\%). Certainly, hematoxylin and eosin staining may have compromised the Se of the histopathology in detecting fatty liver. Alcohol contained in these stains may have dissolved the fat molecules accumulated in the liver samples. A more specific fat staining regimen such Oil Red O and Sudan Black B could have yielded a more precise diagnosis; however, in human and several animal models, the use of hematoxylin and eosin to stain liver samples for the diagnosis of fat infiltration still remains a common practice. Histopathological evaluation of fatty liver is focused on the detection of the typical cytoplasmic vacuoles observed in hepatocytes, regardless of fat removal from the sample processing protocol (Benedict and Zhang, 2017; Lau et al., 2017).

Cytology versus liver TG content yielded the highest level of agreement (kappa coefficient $=0.53$ ). The level of agreement was moderate. However, for cytology versus histopathology and histopathology versus liver TG content, the agreement was fair because the kappa coefficient was between 0.2 and 0.4 (0.35 and 0.37 , respectively; Landis and Koch, 1977).

Supporting our study are the results of the powerful statistical technique of cumulative logit for ordinal data. As the TG content in the liver increases, the likelihood of a higher cytological and histopathological score increases and the likelihood of a lower score decreases. In this analysis, outcome variables were analyzed as they are (ordinal discrete data or scores) as a function of a continuous variable (liver TG content, $\mathrm{mg} / \mathrm{g}$ ).

One of the major drawbacks of this study was the fact that the samples were obtained from livers removed postmortem; therefore, the results cannot be directly extrapolated to samples obtained from a living animal. Sampling of the liver for cytology in a live bovine can present more complications than the study simulated. Trespassing of the skin and muscles with a thin needle can affect the aspiration process of the liver sample. However, the purpose of this pilot study was to evaluate the technique of cytology in an adequately obtained sample. Having already demonstrated that the cytological technique offers acceptable results, the next step will be to conduct studies using live dairy cows and to establish results of the cytological technique in vivo. If the results in live animals proves to be similar to the current investigation, FNAC could have a potential use in clinical practice for the diagnosis of fatty liver in diseased animals, especially in those postpartum dairy cows with liver TG content $>5 \%$. In light of the present results, cytology using livers obtained postmortem and removed from the animal carcass has an acceptable Se and Sp using TG content with a cut-off value of $2 \%$ as a gold standard. However, studies with live animals are warranted to compare the cytological results to the current investigation.

\section{ACKNOWLEDGMENTS}

We thank the students of the College of Veterinary Medicine, University of Missouri (Columbia), for assisting in the editing of this manuscript. This work was supported by the USDA National Institute of Food and Agriculture (Columbia, MO), Animal Health Formula Funds, project 1006957.

\section{REFERENCES}

Arndt, T. P., and S. M. Shelly. 2014. The liver. Pages 354-371 in Diagnostic Cytology and Hematology of the Dog and Cat. 4th ed. A. C. Valenciano and R. L. Cowell, ed. Elsevier/Mosby, Saint Louis, MO.

Benedict, M., and X. Zhang. 2017. Non-alcoholic fatty liver disease: An expanded review. World J. Hepatol. 9:715-732.

Bobe, G., J. W. Young, and D. C. Beitz. 2004. Invited Review: Pathology, etiology, prevention, and treatment of fatty liver in dairy cows. J. Dairy Sci. 87:3105-3124.

Brown, D., A. J. Van Wettere, and J. M. Cullen. 2017. Hepatobiliary system and exocrine pancreas. Pages $412-470$ in Pathologic Basis of Veterinary Disease. 6th ed. J. F. Zachary, ed. Elsevier, St. Louis, MO.

Crookenden, M. A., C. G. Walker, H. Peiris, Y. Koh, A. Heiser, J. J. Loor, K. M. Moyes, A. Murray, V. S. R. Dukkipati, J. K. Kay, S. Meier, J. R. Roche, and M. D. Mitchell. 2016. Short communication: Proteins from circulating exosomes represent metabolic state in transition dairy cows. J. Dairy Sci. 99:7661-7668.

Geelen, M. J. H., and T. Wensing. 2006. Studies on hepatic lipidosis and coinciding health and fertility problems of high-producing dairy cows using the "Utrecht fatty liver model of dairy cows". A review. Vet. Q. 28:90-104.

Gerspach, C., S. Imhasly, M. Gubler, H. Naegeli, M. Ruetten, and E. Laczko. 2017. Altered plasma lipidome profile of dairy cows with fatty liver disease. Res. Vet. Sci. 110:47-59.

Gross, J. J., F. J. Schwarz, K. Eder, H. A. van Dorland, and R. M. Bruckmaier. 2013. Liver fat content and lipid metabolism in dairy cows during early lactation and during a mid-lactation feed restriction. J. Dairy Sci. 96:5008-5017.

Haudum, A., A. Starke, M. Beyerbach, P. Wohlsein, and J. Rehage. 2011. Ultrasonographic assessment of liver dimensions in dairy cows with different hepatic triacylglycerol content. J. Anim. Sci. 89:1392-1400.

Herdt, T. H. 1988. Fatty liver in dairy cows. Vet. Clin. North Am. Food Anim. Pract. 4:269-287.

Herdt, T. H. 2000. Ruminant adaptation to negative energy balance. Influences on the etiology of ketosis and fatty liver. Vet. Clin. North Am. Food Anim. Pract. 16:215-230.

Hoff, B., and J. Cote. 1996. Fine needle aspiration and liver cytology-A simple method for diagnosis and prognosis of fatty liver in cattle. Bov. Pract. 30:53-55.

Jorritsma, R., H. Jorritsma, Y. H. Schukken, P. C. Bartlett, Th. Wensing, and G. H. Wentink. 2001. Prevalence and indicators of post partum fatty infiltration of the liver in nine commercial dairy herds in The Netherlands. Livest. Prod. Sci. 68:53-60.

Kalaitzakis, E., N. Panousis, N. Roubies, N. Giadinis, E. Kaldrymidou, M. Georgiadis, and H. Karatzias. 2010. Clinicopathological 
evaluation of downer dairy cows with fatty liver. Can. Vet. J. $51: 615-622$.

Komemushi, A., S. Kanno, S. Suzuki, A. Sano, S. Kariya, M. Nakatani, R. Y. Yoshida, Y. Kono, Y. Harima, and N. Tanigawa. 2015. Evaluation of an aspiration-type semiautomatic cutting biopsy needle. Minim. Invasive Ther. Allied Technol. 24:250-252.

Landis, J. R., and G. G. Koch. 1977. The measurement of observer agreement for categorical data. Biometrics 33:159-174.

Lau, J. K, X. Zhang, and J. Yu. 2017. Animal models of non-alcoholic fatty liver disease: Current perspectives and recent advances. J. Pathol. 241:36-44.

McCarthy, M. M., M. S. Piepenbrink, and T. R. Overton. 2015. Associations between hepatic metabolism of propionate and palmitate in liver slices from transition dairy cows. J. Dairy Sci. 98:7015-7024.

McCarthy, M. M., T. Yasui, M. J. B. Felippe, and T. R. Overton. 2016. Associations between the degree of early lactation inflammation and performance, metabolism, and immune function in dairy cows. J. Dairy Sci. 99:680-700.

Nalbantoglu, I. L., and E. M. Brunt. 2014. Role of liver biopsy in nonalcoholic fatty liver disease. World J. Gastroenterol. 20:9026-9037.

Petit, H. V., M. F. Palin, and L. Doepel. 2007. Hepatic lipid metabolism in transition dairy cows fed flaxseed. J. Dairy Sci. 90:47804792 .
Rafia, S., T. Taghipour-Bazargani, F. Asadi, A. Vajhi, and S. Bokaie. 2011. Periparturition alterations to liver ultrasonographic echotexture and fat mobilization parameters in clinically healthy Holstein cows. Vet. Res. Commun. 35:531-540.

Rothuizen, J., and D. C. Twedt. 2009. Liver biopsy techniques. Vet. Clin. North Am. Small Anim. Pract. 39:469-480.

Starke, A., S. Schmidt, A. Haudum, T. Scholbach, P. Wohlsein, M. Beyerbach, and J. Rehage. 2011. Evaluation of portal blood flow using transcutaneous and intraoperative Doppler ultrasonography in dairy cows with fatty liver. J. Dairy Sci. 94:2964-2971.

Swecker, W. S. 2014. Trace mineral feeding and assessment. Vet. Clin. North Am. Food Anim. Pract. 30:671-688.

Tharwat, M., A. Takamizawa, Y. Z. Hosaka, D. Endoh, and S. Oikawa. 2012. Hepatocyte apoptosis in dairy cattle during the transition period. Can. J. Vet. Res. 76:241-247.

Weijers, G., A. Starke, J. M. Thijssen, A. Haudum, P. Wohlsein, J. Rehage, and C. L. de Korte. 2012. Transcutaneous vs. intraoperative quantitative ultrasound for staging bovine hepatic steatosis. Ultrasound Med. Biol. 38:1404-1413.

Weiss, D. J., and M. Moritz. 2002. Liver cytology. Vet. Clin. North Am. Small Anim. Pract. 32:1267-1291. 